\title{
Increased prevalence of Autoimmune Diseases in Children with Chronic Spontaneous Urticaria
}

Michelle Le ${ }^{1}$, Lydia Zhang ${ }^{1}$, Sofianne Gabrielli ${ }^{1}$, Connor Prosty ${ }^{1}$, Laura May Miles ${ }^{1}$, Elena Netchiporouk $^{1}$, Sharon Baum ${ }^{2}$, Shoshana Greenberger ${ }^{2}$, LUIS FELIPE ENSINA ${ }^{3}$, Fatemeh Jafarian $^{1}$, Xun Zhang ${ }^{4}$, and Moshe Ben-Shoshan ${ }^{5}$

${ }^{1}$ McGill University Health Centre

${ }^{2}$ Sheba Medical Center

${ }^{3}$ Universidade Federal de Sao Paulo

${ }^{4}$ Research Institute of the McGill University Health Centre

${ }^{5}$ McGill University

November 19, 2021

Increased prevalence of Autoimmune Diseases in Children with Chronic Spontaneous Urticaria

Michelle Le, MD ${ }^{1}$, Lydia Zhang $\mathrm{MD}^{2}$, Sofianne Gabrielli $\mathrm{MSc}^{2}$, Connor Prosty BSc, MD $(\mathrm{c})^{1}$, Laura May Miles LLM, MD(c) ${ }^{2}$, Elena Netchiporouk, MD, MSc ${ }^{1}$, Sharon Baum, MD ${ }^{3}$, Shoshana Greenberger, $\mathrm{MD}^{3}$, Luis F. Ensina, MD, MSc, $\mathrm{PhD}^{4}$, Fatemeh Jafarian, $\mathrm{MD}^{1}$, Xun Zhang, $\mathrm{PhD}^{5}$, Moshe Ben-Shoshan, MD, $\mathrm{MSc}^{2}$

${ }^{1}$ Division of Dermatology, McGill University, Montreal, QC, Canada

${ }^{2}$ Division of Pediatric Allergy and Clinical Immunology, Department of Pediatrics, McGill University Health Centre, Montreal, QC, Canada

${ }^{3}$ Department of Dermatology, Chaim Sheba Medical Center, Tel-Aviv University, Sackler School of Medicine, Tel Hashomer, Israel

${ }^{4}$ Department of Pediatrics, Federal University of São Paolo, Brazil

${ }^{5}$ Centre for Outcome Research and Evaluation, Research Institute of McGill University Health Centre, Montreal, QC, Canada

Correspondence: Michelle Le, M.D., 1001 Decarie Blvd, Montréal, Québec, H4A 3J1 email: michelle.le@mail.mcgill.ca

Article type: Letter

Manuscript word count: 981

References : 10

Tables : 1

Figures: None

Funding sources : None

Conflicts of interest: None declared 
IRB Approval Status : Reviewed and approved by McGill University Health Centre; approval 12-255GEN

\section{Abbreviations/Acronyms:}

AuIDs: autoimmune diseases

CSU: chronic spontaneous urticaria

aOR: adjusted Odds Ratio

complete blood count (CBC)

CRP: C-reactive protein

BAT: Basophil activation tests

To the Editor:

Chronic spontaneous urticaria (CSU) is a disease characterized by the presence of angioedema and/or pruritic transient wheals that occur in the absence of an identifiable triggers and persist for more than 6 weeks. Although the precise etiology of CSU remains unknown, studies have suggested that the majority of cases of CSU in adults are due to autoimmunity ${ }^{1}$. Further, an association of CSU with autoimmune diseases (AuIDs) has been suggested in adult patients, although the prevalence of AuIDs in pediatric patients has not yet been established. A recent meta-analysis reported that the prevalence of autoimmune thyroiditis, pernicious anemia, vitiligo, type I diabetes mellitus, Graves' disease, celiac disease and rheumatoid arthritis were increased among adults with CSU compared to the general population ${ }^{2}$. However, children were excluded from this meta-analysis due to the lack of studies on comorbid AuIDs in CSU patients. We aimed to assess the prevalence of hypothyroidism, lupus, type I diabetes mellitus and juvenile arthritis in a cohort of pediatric patients with CSU compared to the general pediatric population and to identify sociodemographic factors, clinical characteristics and biomarkers associated with increased risk of AuIDs in CSU.

Children with CSU were prospectively recruited at the Montreal Children's Hospital Allergy and Immunology clinic in Montreal, Canada, from 2013 to 2019. A complete medical history and physical examination were performed at study entry and every 3-6 months. Data on patient demographics, co-morbidities (including physician reported diagnosis of AuIDs), and clinical characteristics were collected at study entry through a standardized questionnaire. Families were queried at each follow up visit on the development of new comorbidities including AuIDs.

As part of the standard of care, all patients had a baseline complete blood count (CBC), levels of Creactive protein $(\mathrm{CRP})$, total immunoglobulin $(\mathrm{IgE})$, and tryptase measured. Additional investigations (e.g., antinuclear antibody serologic tests, skin biopsies) were performed only if clinical features were suggestive of autoimmune disease or if the diagnosis of CSU was unclear. Basophil activation tests (BATs) using CD63 marker expression were performed by flow cytometry as previously described by Netchiporouk et al.$^{3}$.

All statistical analyses were done using R version 3.2.2 (R Core Team 2013; R Foundation for Statistical Computing, Vienna, Austria). The Fisher's exact test was used to compare the prevalence of AuIDs in our CSU cohort to the general prevalence of AuIDs in children. Multivariate logistic regression was conducted to determine clinical factors associated with the presence of AuIDs in pediatric CSU.

This study was approved by the McGill University Health Centre Ethics Committee. Written consent was obtained from all participants.

Our analysis included 191 children patients, $49.2 \%$ were males and the median age was 9.4 years [IQR: 4.85, 13.65].

Overall, 10 patients in our cohort reported AuIDs. Specifically, the prevalence of hypothyroidism was $2.10 \%$ $(\mathrm{n}=4)$, type I diabetes $1.57 \%(\mathrm{n}=3)$, juvenile rheumatoid arthritis $1.05 \%(\mathrm{n}=2)$, and lupus $0.52 \%(\mathrm{n}=1)$. These results were compared to published prevalence data of AuIDs in children in Europe. The prevalence 
of hypothyroidism, lupus, juvenile arthritis and type I diabetes were significantly increased in our cohort compared to children in the general population ( $\mathrm{p}<0.005$ for each) (Table 1$)$.

Multivariate logistic regression analyses revealed that hypothyroidism was associated with increased age (adjusted Odds Ratio (aOR):1.01 [95\%CI: 1.01, 1.02]) and increased CD63 levels (aOR:1.00 [95\%CI: 1.00, 1.01]) while adjusting for sex, family history of AuIDs, IgE, tryptase, CRP levels and absolute basophil count.

To the best of our knowledge, this is the first study comparing the prevalence of AuIDs in pediatric CSU patients to the general pediatric population. Our data revealed that AuIDs, such as hypothyroidism, lupus, juvenile arthritis, and type I diabetes, were more common among children with CSU in comparison to the general pediatric population. Additionally, hypothyroidism was found to be associated to increased age and increased CD63 levels.

Although the prevalence of AuIDs was increased among children with CSU, they are nevertheless relatively rare ( $<5 \%$ of children). We therefore recommend assessing for AuIDs only if there is a suggestive clinical history and physical exam. These results and recommendations are more conservative than those published by Kosmeri et al.,(2019) ${ }^{4}$ who indicated that screening for AuIDs would be beneficial as diseases, such as celiac disease, may be diagnosed in the absence of indicative signs ${ }^{4}$.

The higher prevalence of AuIDs in children with CSU is in line with the shift of paradigm regarding the pathogenesis of CSU suggesting it is an autoimmune or auto-allergy rather than an idiopathic condition. It is now thought that CSU is an autoimmune condition caused in most cases by a type I hypersensitivity reaction to various self-antigens that bind to IgE (auto-allergy) $)^{1}$.

Our results suggest an association between autoimmune hypothyroidism among children with CSU and older age and elevated CD63. A review of thyroid disease in adults determined that the likelihood of having an autoimmune thyroid disease increases with age ${ }^{5}$. It is hypothesized that epigenetic changes due to environmental factors, contribute to the development of inflammation and autoimmunity with increasing age ${ }^{5}$. Additionally, CD63 is increasingly being accepted as a marker of IgG mediated autoimmunity in CSU $^{3}$.

There are several limitations in our study. Firstly, as reference prevalence values were not available in children in North America, we compared our estimate to European data that do not necessarily reflect the Canadian pediatric population. Nevertheless, the prevalence of AuIDs in European children is not expected to be lower than that of North America, given that studies suggest a higher prevalence of AuIDs in the general population in Europe versus North America ${ }^{6}$. Hence, we expect that differences observed in our population would have been even higher if compared to the Canadian population.

In conclusion, our study suggests that AuIDs is more common among children with CSU and that hypothyroidism is more likely in older children with higher CD63 levels. Increased awareness and appropriate diagnosis of AuIDs when suggested on clinical history or physical exam will contribute to better management of CSU patients.

Keywords: Chronic spontaneous urticaria, pediatrics, management

Michelle Le, $\mathrm{MD}^{1}$, Lydia Zhang $\mathrm{MD}^{2}$, Sofianne Gabrielli $\mathrm{MSc}^{2}$, Connor Prosty BSc, MD(c) ${ }^{1}$, Laura May Miles LLM, MD(c) $)^{2}$, Elena Netchiporouk, MD, $\mathrm{MSc}^{1}$, Sharon Baum, $\mathrm{MD}^{3}$, Shoshana Greenberger, $\mathrm{MD}^{3}$, Luis F. Ensina, MD, MSc, $\mathrm{PhD}^{4}$, Fatemeh Jafarian, $\mathrm{MD}^{1}$, Xun Zhang, PhD ${ }^{5}$, Moshe Ben-Shoshan, MD, $\mathrm{MSc}^{2}$

${ }^{1}$ Division of Dermatology, McGill University, Montreal, QC, Canada

${ }^{2}$ Division of Pediatric Allergy and Clinical Immunology, Department of Pediatrics, McGill University Health Centre, Montreal, QC, Canada

${ }^{3}$ Department of Dermatology, Chaim Sheba Medical Center, Tel-Aviv University, Sackler School of Medicine, Tel Hashomer, Israel 
${ }^{4}$ Department of Pediatrics, Federal University of São Paolo, Brazil

${ }^{5}$ Centre for Outcome Research and Evaluation, Research Institute of McGill University Health Centre, Montreal, QC, Canada

REFERENCES

1. Schmetzer O, Lakin E, Topal FA, et al. IL-24 is a common and specific autoantigen of $\operatorname{IgE}$ in patients with chronic spontaneous urticaria.J Allergy Clin Immunol . Sep 2018;142(3):876-882. doi:10.1016/j.jaci.2017.10.035

2. Kolkhir P, Borzova E, Grattan C, Asero R, Pogorelov D, Maurer M. Autoimmune comorbidity in chronic spontaneous urticaria: A systematic review. Autoimmunity Reviews . 2017/12/01/ 2017;16(12):1196-1208. doi:https://doi.org/10.1016/j.autrev.2017.10.003

3. Netchiporouk E, Moreau L, Rahme E, Maurer M, Lejtenyi D, Ben-Shoshan M. Positive CD63 Basophil Activation Tests Are Common in Children with Chronic Spontaneous Urticaria and Linked to High Disease Activity.Int Arch Allergy Immunol . 2016;171(2):81-88. doi:10.1159/000451084

4. Kosmeri C, Siomou E, Challa A, Tsabouri S. Investigation of Autoimmune Disease in Children with Chronic Spontaneous Urticaria.International Archives of Allergy and Immunology . 2019;180(4):250-254. doi:10.1159/000502521

5. McLeod DS, Cooper DS. The incidence and prevalence of thyroid autoimmunity. Endocrine . Oct 2012;42(2):252-65. doi:10.1007/s12020-012-9703-2

6. Danchenko N, Satia JA, Anthony MS. Epidemiology of systemic lupus erythematosus: a comparison of worldwide disease burden. Lupus . 2006;15(5):308-18. doi:10.1191/0961203306lu2305xx

7. Hunter I, Greene SA, MacDonald TM, Morris AD. Prevalence and aetiology of hypothyroidism in the young. Arch Dis Child . Sep 2000;83(3):207-10. doi:10.1136/adc.83.3.207

8. Fatoye F, Gebrye T, Svenson LW. Real-world incidence and prevalence of systemic lupus erythematosus in Alberta, Canada. Rheumatol Int . Sep 2018;38(9):1721-1726. doi:10.1007/s00296-018-4091-4

9. Shiff NJ, Oen K, Kroeker K, Lix LM. Trends in Population-Based Incidence and Prevalence of Juvenile Idiopathic Arthritis in Manitoba, Canada. Arthritis Care Res (Hoboken). Mar 2019;71(3):413-418. doi:10.1002/acr.23606

10. Dabelea D, Mayer-Davis EJ, Saydah S, et al. Prevalence of type 1 and type 2 diabetes among children and adolescents from 2001 to 2009.Jama . May 7 2014;311(17):1778-86. doi:10.1001/jama.2014.3201

\section{TABLES}

Table 1. Comparing the proportion of AuIDs in the general pediatric population to the proportion of AuIDs in pediatric CSU using the Fisher's Exact test.

\begin{tabular}{llll}
\hline & $\begin{array}{l}\text { Reference proportions } \\
\text { of AuIDs in the } \\
\text { general pediatric } \\
\text { population (\%) }\end{array}$ & $\begin{array}{l}\text { Proportions of AuIDs } \\
\text { from our cohort of } \\
\text { CSU patients (\%) }\end{array}$ & $\begin{array}{l}\text { P-value, 95\% } \\
\text { Confidence interval }\end{array}$ \\
\hline Autoimmune disease & $140 / 103,500(0.135 \%)^{7}$ & $4 / 191(2.10 \%)$ & $\begin{array}{l}\text { P-value }=0.00017(4.19, \\
42.01)\end{array}$ \\
Hypothyroidism & $43 / 852,190(0.005 \%)^{8}$ & $1 / 191(0.52 \%)$ & $\begin{array}{l}\text { P-value }=0.00981(2.57, \\
629.2) \\
\text { Lupus }\end{array}$ \\
Juvenile Arthritis & $52.86 / 100,000(0.053 \%)^{9}$ & $2 / 191(1.05 \%)$ & $76.8)$
\end{tabular}




\begin{tabular}{llll}
\hline & $\begin{array}{l}\text { Reference proportions } \\
\text { of AuIDs in the } \\
\text { general pediatric } \\
\text { population (\%) }\end{array}$ & $\begin{array}{l}\text { Proportions of AuIDs } \\
\text { from our cohort of } \\
\text { CSU patients (\%) }\end{array}$ & $\begin{array}{l}\text { P-value, 95\% } \\
\text { Confidence interval }\end{array}$ \\
\hline Tutoimmune disease & $1.93 / 1,000(0.19 \%)^{10}$ & $3 / 191(1.57 \%)$ & $\begin{array}{l}\text { P-value }=0.0317(0.903, \\
95.7)\end{array}$ \\
\hline
\end{tabular}

